\title{
Electromagnetic Waves in an Inhomogeneous Anisotropic Medium of the Model Ionosphere
}

\author{
Masayoshi MAmbo and Isamu NAGANo \\ Department of Electrical Engineering, Faculty of Technology, \\ Kanazawa University, Kanazawa, Japan
}

(Received September 18, 1972; Revised July 2, 1973)

\begin{abstract}
Electromagnetic fields of a wave propagating in the ionospheric $D$ region with its wave normal directing vertically upwards can be described by the Weber function. The model ionosphere pertinent to the present investigation is composed of the parabolic electron density profile with a constant collision frequency and the vertical magnetic field. The distribution of wave electric field intensity, reflection, transmission and conversion coefficients are numerically calculated. It is shown that the reflection coefficient for the right handed circularly polarized wave has a spirally diminishing characteristic in the amplitude-phase polar coordinate plane for increasing wave frequency. The left handed circularly polarized wave is reflected below the altitude where the wave frequency approaches to the penetration frequency. It is also found that the conversion coefficient of the transmitted wave decreases abruptly above a specific value of collision frequency.
\end{abstract}

\section{Introduction}

The plasma condition for LF radio waves in the ionosphere or in the re-entry region of a spacecraft is inhomogeneous and anisotropic. As it is difficult to treat analytically the behaviour of waves in an inhomogeneous anisotropic medium by the method of full wave theory, most of ealier investigations have been made by utilizing the ray theory. There have been some attempts, recently, to solve the problem by full wave theory for an ionosphere of homogeneous anisotropic or inhomogeneous isotropic medium (BUDden, 1961; Galejs and Row, 1964; Mambo and Shimakura, 1971). By applying the full wave computer method, Pitteway (1965) has studied the electromagnetic fields inside a horizontally stratified ionosphere for a radio wave incident from below. On the other hand, KozAKi and Mushiake (1968) have obtained analytically the intensity distribution of electromagnetic waves, reflection and conversion coefficients in the model ionosphere, in which the collision is neglected. There has been no attempt to solve analytically the electromagnetic fields, the reflection and transmission coefficients in the inhomogeneous anisotropic ionosphere which includes the effect of collision. 
In the present paper, we investigate the above problem for the case of a radio wave incident at vertical angle to the model ionosphere in which the electron density profile is assumed to be of parabolic, the collision is constant throughout the layer and an external static magnetic field assumed to be vertical. The wave electric field intensity, reflection, transmission and conversion coefficients are derived directly from the basic equations in a plasma and they are numerically calculated by using the value of the ionospheric $D$ layer (BouRDEAU et al., 1965, 1966). Discussions are also made on the effect of collision, wave reflection and transmission characteristics and the transition from the anisotropic to the isotropic medium of the ionosphere.

\section{Basic Equations and Solutions}

Let us consider an inhomogeneous anisotropic ionosphere in the region between $z=0$ and $z=z_{h}$ (Fig. 1). With field variables which vary with time as

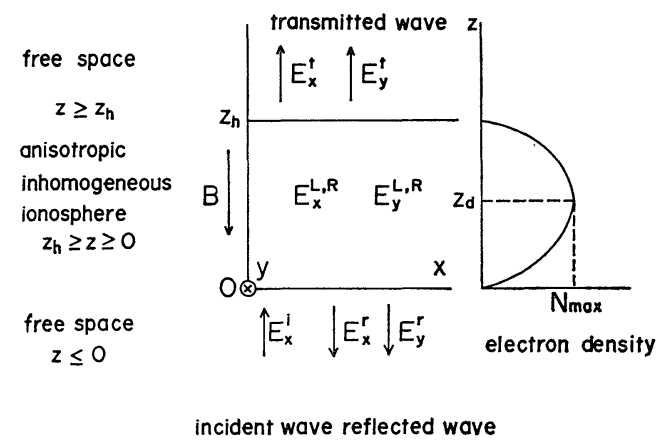

Fig. 1. Electromagnetic fields in each region and electron density profile.

$e^{j \omega t}$, electromagnetic wave fields in the plasma satisfy the following Maxwell equations;

$$
\begin{aligned}
& \nabla \times H=j \omega \varepsilon_{0}[\kappa] E, \\
& \nabla \times E=-j \omega \mu_{0} H,
\end{aligned}
$$

where $\varepsilon_{0}$ and $\mu_{0}$ are the dielectric constant and permeability pertaining to free space. Suppose that the external magnetic field is vertically downward, the relative tensor dielectric constant $[\kappa]$ which is an analytic function of the altitude $z$ is given by

$$
[\kappa]=\left[\begin{array}{ccc}
\kappa_{1} & j \kappa_{2} & 0 \\
-j \kappa_{2} & \kappa_{1} & 0 \\
0 & 0 & \kappa_{3}
\end{array}\right],
$$


where

$$
\begin{gathered}
\kappa_{1}=1-\frac{X(z) U}{U^{2}-Y^{2}}, \quad \kappa_{2}=\frac{X(z) Y}{U^{2}-Y^{2}}, \quad \kappa_{3}=1-\frac{X(z)}{U}, \\
X(z)=\frac{N(z) e^{2}}{m \varepsilon_{0} \omega^{2}}, \quad U=1-j Z, \quad Y=\frac{e B}{m \omega}, \quad Z=\frac{\nu}{\omega},
\end{gathered}
$$

$\omega:$ wave angular frequency $[\mathrm{rad} / \mathrm{sec}]$,

$m, e$ : mass [kg] and charge [C] of an electron,

$\nu$ : mean collision frequency of electron $[\mathrm{Hz}]$,

$B$ : flux density of the external magnetic field $\left[\mathrm{Wb} / \mathrm{m}^{2}\right]$,

$N(z)$ : electron density profile [number $/ \mathrm{m}^{3}$ ].

Since the geometry is one dimensional, $\partial / \partial x=0, \partial / \partial y=0$ in Eqs. (1) and (2). Then, electric field components $E_{x}$ and $E_{y}$ satisfy the following simultaneous differential equations:

$$
\begin{aligned}
& \frac{\mathrm{d}^{2} E_{x}}{\mathrm{~d} z^{2}}+k_{0}^{2} \kappa_{1} E_{x}=-j k_{0} \kappa_{2} E_{y}, \\
& \frac{\mathrm{d}^{2} E_{y}}{\mathrm{~d} z^{2}}+k_{0}^{2} \kappa_{1} E_{y}=j k_{0} \kappa_{2} E_{x},
\end{aligned}
$$

where $k_{0}$ is the propagation constant in free space.

As both Eqs. (4) satisfy

$$
E_{y}=\mp j E_{x}
$$

this shows that there are two circularly polarized waves of the opposite rotation in the case of the vertical magnetic field and of normal incidence. Substituting Eq. (5) in Eq. (4), we get

$$
\frac{\mathrm{d}^{2} E_{x}}{\mathrm{~d} z^{2}}+k^{2} E_{x}=0
$$

where

$$
k^{2}=k_{0}^{2}\left(1-\frac{X(z)}{U \pm Y}\right) .
$$

The plus sign in the above equation corresponds to $E_{y} / E_{x}=-j$, and the minus $\operatorname{sign} E_{y} / E_{x}=+j$. The electric vectors rotate, respectively, in anticlockwise and clockwise to an observer looking toward the external magnetic field, i.e., left handed and right handed circular polarizations, which are denoted as $\mathrm{L}$ and $\mathrm{R}$ waves, respectively.

In the present model of the ionosphere, the electron density is given by the following formula:

$$
N(z)=N_{\max } \frac{z\left(2 z_{\mathrm{d}}-z\right)}{z_{\mathrm{d}}^{2}},
$$

$N_{\max }:$ maximum electron density $\left[\right.$ number $\left./ \mathrm{m}^{3}\right]$,

$z_{\mathrm{d}}$ : altitude of the maximum electron density $[\mathrm{m}]$. 
From Eqs. (6) and (8),

$$
\frac{\mathrm{d}^{2} E_{x}}{\mathrm{~d} z^{2}}+\left(k_{0}^{2}\left(1-\frac{X_{\mathrm{m}}}{U \pm Y}\right)+k_{0}^{2} \frac{X_{\mathrm{m}}}{U \pm Y}\left(\frac{z-z_{\mathrm{d}}}{z_{\mathrm{d}}}\right)^{2}\right) E_{x}=0,
$$

where $X_{\mathrm{m}}$ is the maximum value of $X$, corresponding to $N=N_{\max }$. Now let

$$
\begin{gathered}
\xi=\left(\frac{4 k_{0}^{2} X_{\mathrm{m}}}{U \pm Y}\right)^{\frac{1}{4}}\left(z-z_{\mathrm{d}}\right) e^{-j(\pi / 4)} \\
\lambda=\left(\frac{4 k_{0}^{2} X_{\mathrm{m}}}{(U \pm Y) z_{\mathrm{d}}^{2}}\right)^{-\frac{1}{2}} k_{0}^{2}\left(1-\frac{X_{\mathrm{m}}}{U \pm Y}\right) e^{j(\pi / 2)}-\frac{1}{2} .
\end{gathered}
$$

Then, Eq. (9) is written as

$$
\frac{\mathrm{d}^{2} E_{x}}{\mathrm{~d} \xi^{2}}+\left(\lambda+\frac{1}{2}-\frac{\xi^{2}}{4}\right) E_{x}=0
$$

which is known as the Weber's differential equation.

Fundamental solutions of Weber's equation are $D_{\lambda}(\xi), D_{\lambda}(-\xi), D_{-\lambda-1}(j \xi)$ and $D_{-\lambda-1}(-j \xi)$. We may chose $D_{\lambda}(\xi)$ and $D_{-\lambda-1}(-j \xi)$ among these by considering the variation of $\xi$ (Fig. 2), since the asymptotic expansion of Weber function has a simple form when its argument is in the range $-\frac{3}{4} \pi$ to $\frac{3}{4} \pi$ (WHITTAKER and WAtson, 1965). Electromagnetic wave fields are given as follows:

$$
\begin{aligned}
& E_{x}=A D_{\lrcorner}(\xi)+B D_{-\lambda-1}(-j \xi), \\
& E_{y}=\mp j E_{x}, \quad H_{x}=\frac{1}{j \omega \mu_{0}} \frac{\mathrm{d} E_{y}}{\mathrm{~d} z}, \quad H_{y}=\frac{-1}{j \omega \mu_{0}} \frac{\mathrm{d} E_{x}}{\mathrm{~d} z},
\end{aligned}
$$

where $E_{x}$ represents $E_{x}^{\mathrm{L}}$ and $E_{x}^{\mathrm{R}}$ for the selection of \pm sign in Eq. (9).

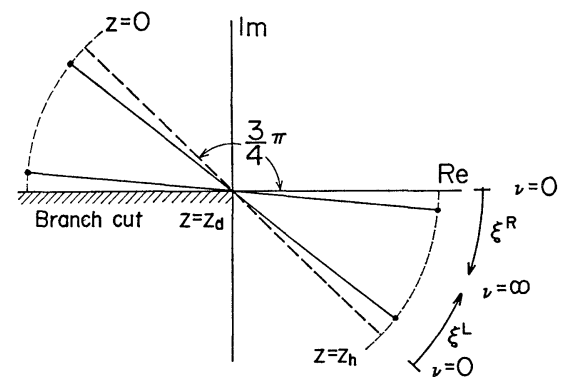

Fig. 2. Variations of $\xi^{\mathrm{L}}$ and $\xi^{\mathrm{R}}$ with height. $\xi^{\mathrm{L}}$ and $\xi^{\mathrm{R}}$ rotate respectively from the dotted line and real axis to meet each other when $\nu$ increases from zero to infinity.

The normal incident plane wave is linearly polarized with its electric vector parallel to the $x$ axis. The electric fields in free space are given by

$$
E_{x}^{i}=E_{0} e^{-j k_{0} z}, \quad E_{x}^{r}=E_{0 x}^{r} e^{j k_{0} z}, \quad E_{y}^{r}=E_{0 y}^{r} e^{j k_{0} z}, \quad \text { with } z<0,
$$


where the incident and reflected waves are denoted by superscript $i$ and $r$, respectively. Similarly the transmitted waves are given by

$$
E_{x}^{\mathrm{t}}=E_{0 x}^{\mathrm{t}} e^{-j k_{0} z}, \quad E_{y}^{\mathrm{t}}=E_{0 y}^{\mathrm{t}} e^{-j k_{0} z}, \quad \text { with } z>z_{h} .
$$

The boundary conditions at $z=0$ and $z=z_{h}$ are;

$$
\begin{aligned}
& \left.\begin{array}{rlrl}
E_{x}^{\mathrm{i}}+E_{x}^{\mathrm{r}} & =E_{x}, & E_{y}^{\mathrm{r}} & =E_{y}, \\
H_{x}^{\mathrm{r}} & =H_{x}, & H_{y}^{\mathrm{i}}+H_{y}^{\mathrm{r}} & =H_{y},
\end{array}\right\} \quad \text { with } z=0, \\
& \left.\begin{array}{ll}
E_{x}=E_{x}^{\mathrm{t}}, & E_{y}=E_{y}^{\mathrm{t}}, \\
H_{x}=H_{x}^{\mathrm{t}}, & H_{y}=H_{y}^{\mathrm{t}},
\end{array}\right\} \quad \text { with } z=z_{h} .
\end{aligned}
$$

The unknown coefficients $A^{\mathrm{L}, \mathrm{R}}, B^{\mathrm{L}, \mathrm{R}}, E_{0 x}^{\mathrm{r}}, E_{0 y}^{\mathrm{r}}, E_{0 x}^{\mathrm{t}}$ and $E_{0 y}^{\mathrm{t}}$ can be determined from eight simultaneous equations in Eq. (14).

Since the reflected wave in free space is composed of left and right handed circularly polarized waves, we get

$$
\begin{aligned}
& E_{x}^{\mathrm{r}}=E_{0 x}^{\mathrm{r}} e^{j k_{0} z}=\left(E_{x}^{\mathrm{r}, \mathrm{L}}+E_{x}^{\mathrm{r}, \mathrm{R}}\right) e^{j k_{0} z}, \\
& E_{y}^{\mathrm{r}}=E_{0 y}^{\mathrm{r}} e^{j k_{0} z}=\left(-j E_{x}^{\mathrm{r}, \mathrm{L}}+j E_{x}^{\mathrm{r}, \mathrm{R}}\right) e^{j k_{0} z} .
\end{aligned}
$$

The reflected wave for left and right handed circular polarizations denoted by the superscripts $r, L$ and $r, R$, respectively, can be determined from Eq. (15) as follows:

$$
\begin{array}{ll}
E_{x}^{\mathrm{r}, \mathrm{L}}=\frac{1}{2}\left(E_{0 x}^{\mathrm{r}}+j E_{0 y}^{\mathrm{r}}\right), & E_{x}^{\mathrm{r}, \mathrm{R}}=\frac{1}{2}\left(E_{0 x}^{\mathrm{r}}-j E_{0 y}^{\mathrm{r}}\right), \\
E_{y}^{\mathrm{r}, \mathrm{L}}=\frac{1}{2}\left(-j E_{0 x}^{\mathrm{r}}+E_{0 y}^{\mathrm{r}}\right), & E_{y}^{\mathrm{r}, \mathrm{R}}=\frac{1}{2}\left(j E_{0 x}^{\mathrm{r}}+E_{0 y}^{\mathrm{r}}\right) .
\end{array}
$$

In the same way, the transmitted waves for left and right handed circular polarization are given by

$$
\begin{array}{ll}
E_{x}^{\mathrm{t}, \mathrm{L}}=\frac{1}{2}\left(E_{0 x}^{\mathrm{t}}+j E_{0 y}^{\mathrm{t}}\right), & E_{x}^{\mathrm{t}, \mathrm{R}}=\frac{1}{2}\left(E_{0 x}^{\mathrm{t}}-j E_{0 y}^{\mathrm{t}}\right), \\
E_{y}^{\mathrm{t}, \mathrm{L}}=\frac{1}{2}\left(-j E_{0 x}^{\mathrm{t}}+E_{0 y}^{\mathrm{t}}\right), & E_{y}^{\mathrm{t}, \mathrm{R}}=\frac{1}{2}\left(j E_{0 x}^{\mathrm{t}}+E_{0 y}^{\mathrm{t}}\right) .
\end{array}
$$

Then, the reflection and transmission coefficients for circular polarization waves are defined as

$$
\mathscr{R}^{\mathrm{L}}=\frac{E_{x}^{\mathrm{r}, \mathrm{L}}}{E^{\mathrm{i}}}, \quad \mathscr{R}^{\mathrm{R}}=\frac{E_{x}^{\mathrm{r}, \mathrm{R}}}{E^{\mathrm{i}}}, \quad \mathscr{T}^{\mathrm{L}}=\frac{E_{x}^{\mathrm{t}, \mathrm{L}}}{E^{\mathrm{i}}}, \quad \mathscr{T}^{\mathrm{R}}=\frac{E_{x}^{\mathrm{t}, \mathrm{R}}}{E^{\mathrm{i}}} .
$$

The ratios of each electric components to incident wave are also defined as follows:

$$
\mathscr{R}^{\mathrm{r}}=\frac{E_{x}^{\mathrm{r}}}{E^{\mathrm{i}}}, \quad \mathscr{R}^{\mathrm{c}}=\frac{E_{y}^{\mathrm{r}}}{E^{\mathrm{i}}}, \quad \mathscr{T}^{\mathrm{t}}=\frac{E_{x}^{\mathrm{t}}}{E^{\mathrm{i}}}, \quad \mathscr{T}^{\mathrm{c}}=\frac{E_{y}^{\mathrm{t}}}{E^{\mathrm{i}}},
$$

where the reflection, transmission and conversion coefficients are denoted by superscripts $r, t$ and $c$, respectively.

3. Numerical Results of $E_{x}, \mathscr{R}$ and $\mathscr{T}$

Numerical results of computed $\left|E_{x}^{\mathrm{L}, \mathrm{R}}\right| / E^{\mathrm{i}}$ are illustrated in Fig. 3, with the 


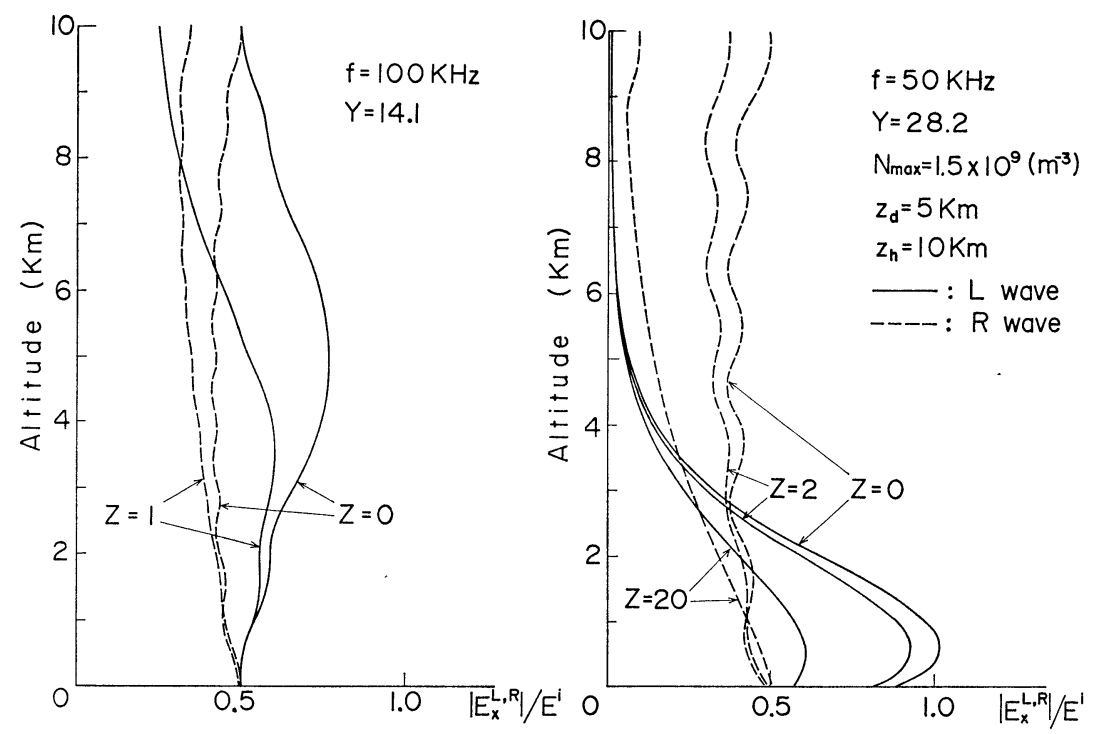

Fig. 3. Distribution of electric field intensity for $\mathrm{R}$ and $\mathrm{L}$ waves.

collision frequency as a parameter. The ionospheric parameters used are $B=$ $5.0 \times 10^{-5} \mathrm{~Wb} / \mathrm{m}^{2}, N_{\max }=1.5 \times 10^{9} \mathrm{~m}^{-3}$ and $z_{h}=10 \mathrm{~km}$. The value of field intensity is normalized by the incident wave $E^{\mathrm{i}}$. It is shown that, in general, the $\mathrm{L}$ wave intensity profile has a peak in the altitude range $0<z \leqq z_{\mathrm{d}}$. The $\mathrm{R}$ wave intensity profile demonstrates clearly a wavy pattern, which is due to wave interferences in the ionosphere. The wave length of this standing wave pattern

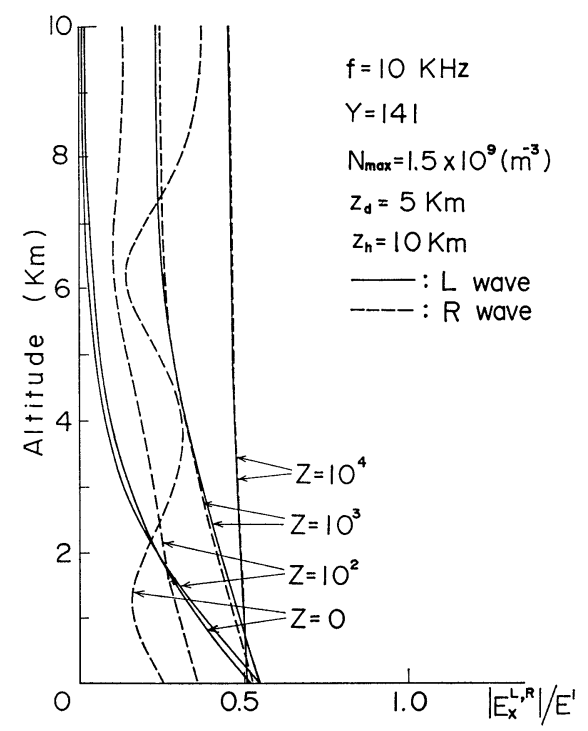

Fig. 4. Distribution of the electric field intensity for large $Z$ values. 

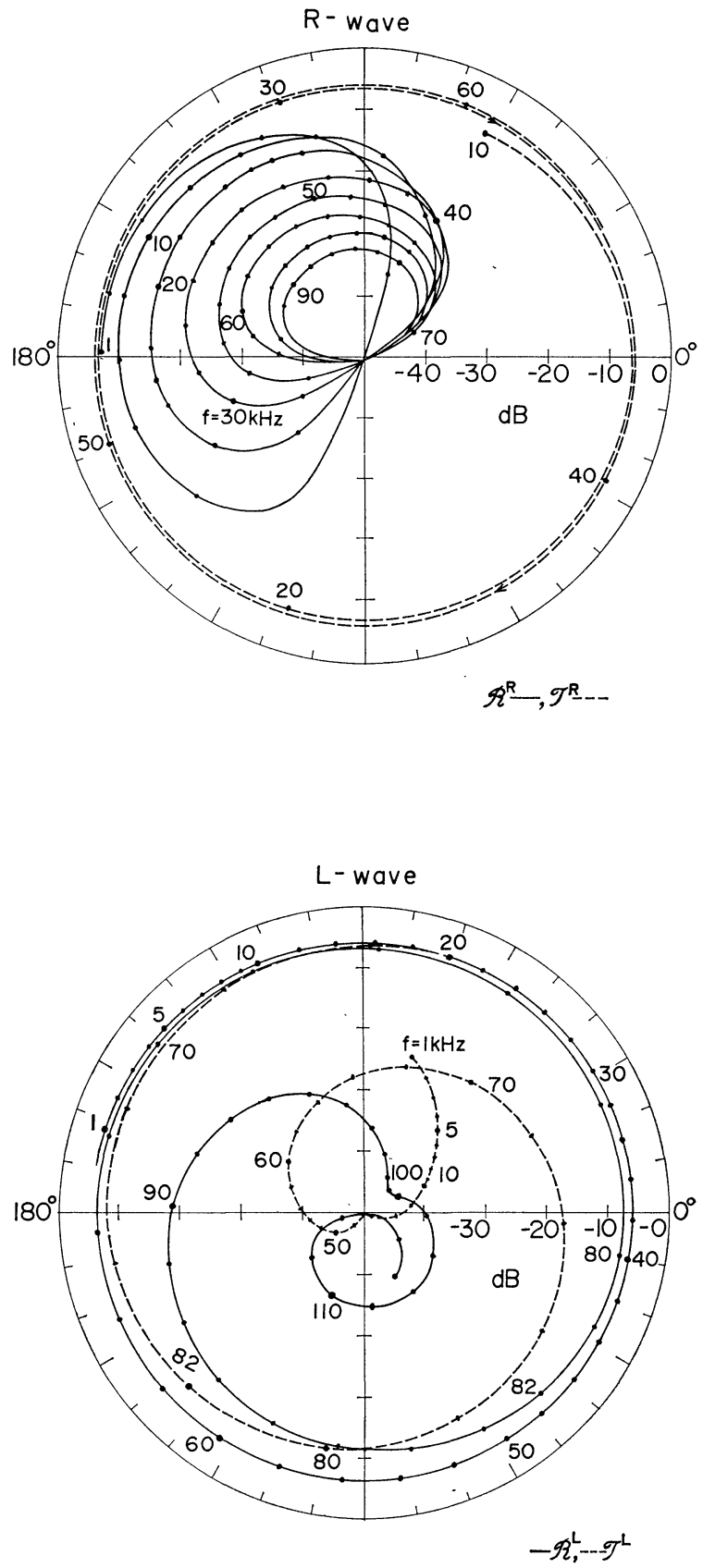

${ }_{\nu}=0, N_{\max }=15 \times 10^{2}\left(\mathrm{~m}^{-3}\right), z_{\mathrm{n}}=10 \mathrm{Km}$

Fig. 5. Variations of reflection and transmission coefficients for $\mathrm{R}$ and $\mathrm{L}$ waves. 
increases as the frequency deceases. The effect of large $Z$ values is shown in Fig. 4. It is evident that the propagation constant $k$ approaches to $k_{0}$ in free space, when $Z$ increase to infinity. The altitude $z$ which satisfies $k^{\mathrm{L}}(z)=0$ for $Z=0$ can be obtained from Eq. (7) as

$$
z_{0}=z_{\mathrm{d}}\left(1 \pm \frac{1}{\sqrt{X_{\mathrm{m}}}} \sqrt{X_{\mathrm{m}}-(1+Y)}\right)=z_{1} \text { or } z_{2} .
$$

In the present model, $z_{0}$, whose $z_{1}$ corresponds to the total reflection point of ray theory, is calculated as:

\begin{tabular}{llll}
\hline$f(\mathrm{kHz})$ & 80 & 50 & 10 \\
\hline$z_{1}(\mathrm{~km})$ & 4.33 & 1.85 & 0.30 \\
\hline$z_{2}(\mathrm{~km})$ & 5.67 & 8.15 & 9.70 \\
\hline
\end{tabular}

The phases of the field of Eq. (13) indicates evanescent in the region from $z_{1}$ to $z_{2}$ (Mambo and NaGANo, 1971).

The behaviour of the reflection and transmission coefficients for varying the wave frequency with $Z=0$ is illustrated in Fig. 5. For $\mathrm{R}$ mode there is no total reflection point, and the wave is mostly transmitted through the ionosphere. The amplitude of the transmission coefficient $\mathscr{T}^{\mathrm{R}}$ is 0.5 or $-6 \mathrm{~dB}$. The reflection coefficient $\mathscr{R}^{\mathrm{R}}$ decreases with increasing frequency. Since $\left|\left(1 / n^{2}\right)(\partial n / \partial z)\right| \ll k_{0}$ where $n$ is refractive index in the layer for $\mathrm{R}$ mode, the medium varies very slowly and there is no appreciable partial reflection inside the layer. Reflection occurs largely at both upper and lower boundaries. The behaviour for L mode is somewhat different. The transmission coefficient decreases drastically below the penetration frequency, which is defined as the wave frequency at which $k^{\mathrm{L}}$ is zero at the point of $N_{\max }$. This penetrating frequency for the $\mathrm{L}$ wave is $f_{t}=$ $82 \mathrm{kHz}$ in the present model. If the ionosphere is loss free $\nu=0$, the some of the energy in the reflected and transmitted waves satisfies $\left|\mathscr{R}^{\mathrm{L}}\right|^{2}+\left|\mathscr{T}^{\mathrm{L}}\right|^{2}=0.5$.

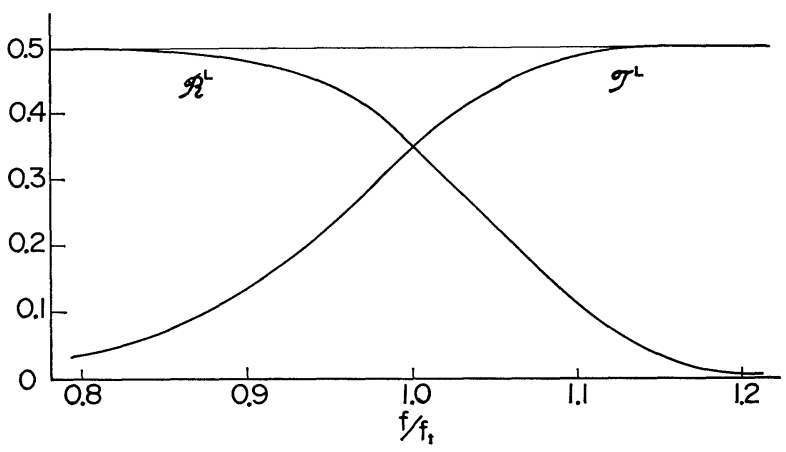

Fig. 6. Reflection coefficient $\mathscr{R}^{\mathrm{L}}$ and transmission coefficient $\mathscr{T}^{\mathrm{L}}$ near the penetration frequency. 
$\mathscr{R}^{\mathrm{L}}$ and $\mathscr{T}^{\mathrm{L}}$ indicate $-9 \mathrm{~dB}(1 / 2 \sqrt{2})$ at $f=f_{t}$, a half of the incident energy of the $\mathrm{L}$ wave being reflected. The transmission near the penetration frequency is shown in Fig. 6.

The variation of $\mathscr{R}^{\mathrm{R}}$ and $\mathscr{T}^{\mathrm{R}}$ for $\nu=2 \pi \times 10^{6}$ is shown in Fig. 7. Attenuating strongly by the collision, the wave is less penetrated through the layer. The reflection vanishes at higher frequencies and the spiral form disappears.

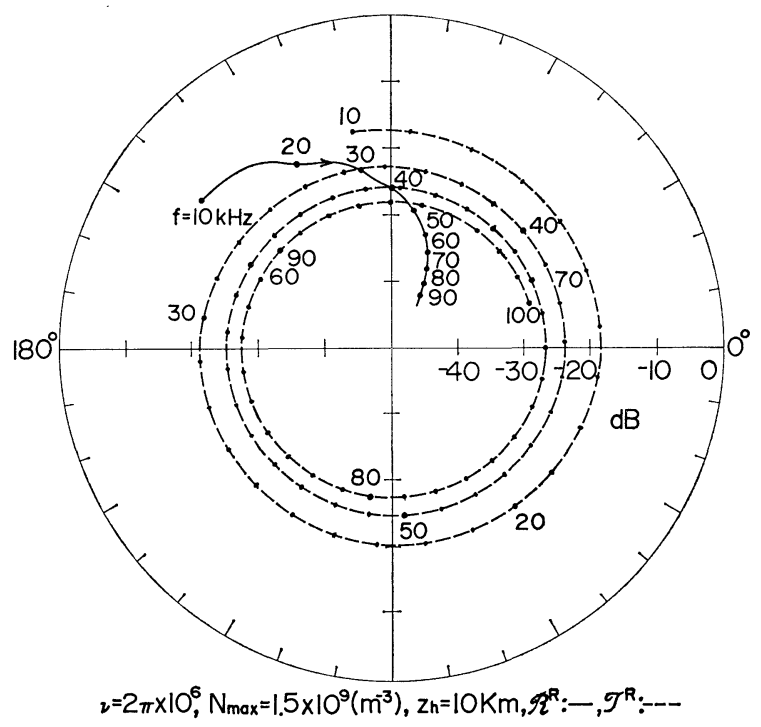

Fig. 7. Variations of reflection and transmission coefficients for right handed circularly polarized wave.

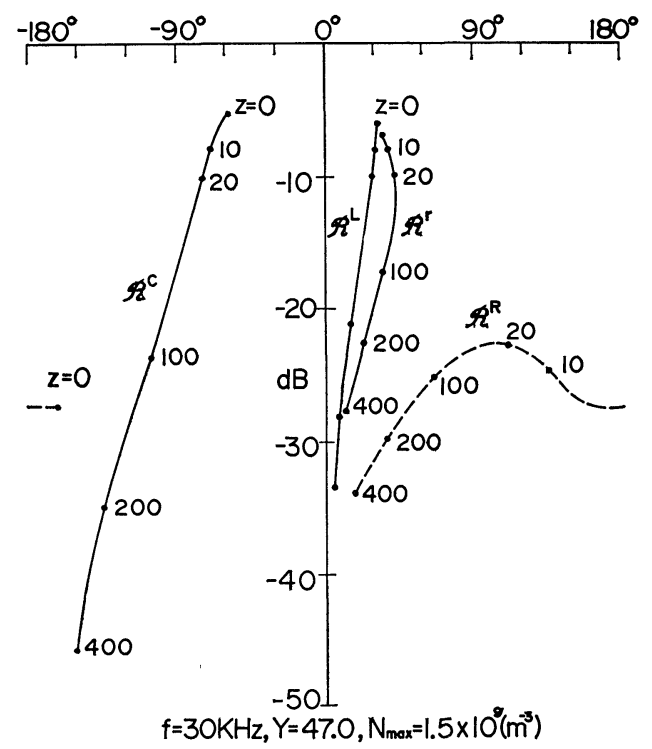

Fig. 8. Variations of reflection and conversion coefficients with $Z$. 
Reflection coefficients are shown in Fig. 8 with the collision term $Z$ as a parameter. Generally, the amplitudes of $\mathscr{R}^{\mathrm{R}}, \mathscr{R}^{\mathrm{L}}, \mathscr{R}^{\mathrm{r}}$ and $\mathscr{R}^{\mathrm{c}}$ decrease as $Z$ increases. However, in certain frequency ranges, $\mathscr{R}^{\mathrm{R}}$ has a maximum at $Z \neq 0$. This may be explained as follows. As stated above, $\mathscr{R}^{\mathrm{R}}$ at $Z=0$ in Fig. 5 is the resultant of the reflected waves from the upper and lower boundaries which are denoted by $\mathscr{R}_{u}^{\mathrm{R}}$ and $\mathscr{R}_{d}^{\mathrm{R}}$ respectively, and are shown in Fig. 9.

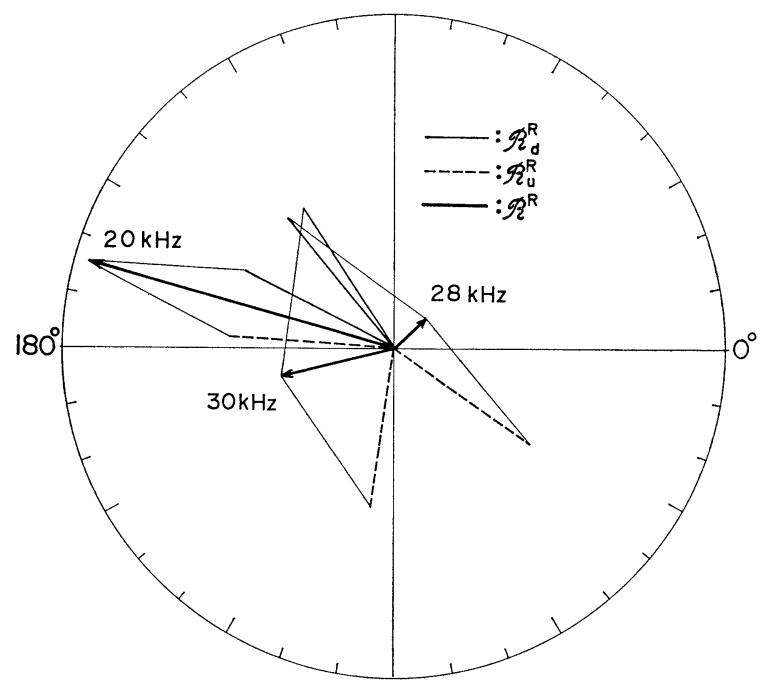

Fig. 9. Separation of reflection component right handed circularly polarized wave.

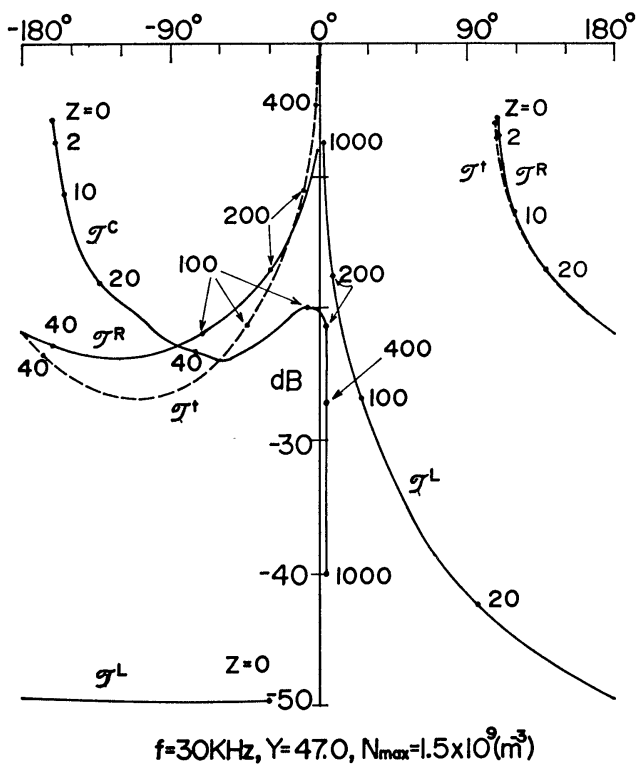

Fig. 10. Variations of transmission and conversion coefficients with $Z$. 
$\mathscr{R}_{u}^{\mathrm{R}}$ shown in Fig. 9 attenuates for increasing $\nu$, therefore the phase of the resultant vector approaches to the phase of the $\mathscr{R}_{d}^{\mathrm{R}}$ so that the phase rotates toward the clockwise direction. $\mathscr{R}_{d}^{\mathrm{R}}$ or $\mathscr{R}^{\mathrm{R}}$ attenuates heavily for further more increasing $\nu$. Then $\mathscr{R}^{\mathrm{R}}$ has a maximum value at $Z \neq 0$. Such variation has also been noted in an inhomogeneous isotropic medium (MАмво and SHIMAKURA, 1971).

Transmission coefficients are shown in Fig. 10 with the collision term $Z$ as a parameter. For $Y \gg 1$, Eq. (7) is

$$
k^{2}=k_{0}^{2}\left(1+\frac{X(z)}{\mp Y+j Z}\right) \text {. }
$$

When $Z \gg Y, k^{2}$ becomes to be independent of $Y$, the medium becomes to be isotropic and $\mathscr{T}^{\mathrm{C}} \rightarrow 0$. Since $Z \gg 1, k^{2} \fallingdotseq k_{0}^{2}$, that is, the medium becomes to be free space and there is no reflection from the model ionosphere. It is interesting to note that the amplitude of $\mathscr{T}^{\mathrm{C}}$ decreases abruptly at $Z \geqq 100-200$. It is found that this value of $Z$ is such that $Z \geq 4 Y$. This suggests that the above condition may represent the transition from anisotropic to isotropic nature of the medium.

We wish to express our sincere appreciation to Professor I. Kimura for his suggestion on this investigation. The computations are performed by using the electric computer FACOM 230-35 in the data processing center, Kanazawa University.

\section{REFERENCES}

Bourdeau, R.E., A.C. Aikin and J.L. Donley, "The lower ionosphere at solar minimum," Goddard Space Flight Center, Ionospheric Physics Reprint Series, X-615-65-304, 1965.

Bourdeau, R.E., A.C. Aikin and J.L. Donley, "Lower ionosphere at solar minimum," $J$. Geophys. Res., 71, 727-740, 1966.

Budden, K.G., "'Radio wave in the ionosphere," Cambridge University Press, London, 1961.

GALEJS, J. and R.V. Row, "Propagation of ELF wave below an inhomogeneous anisotropic ionosphere," IEEE Trans. Ap-12, p. 74, Jan., 1964.

Kozaki, S. and Y. Mushiake, Radio wave propagation in longitudinally inhomogeneous anisotropic medium, J.I.E.C.E. of Japan, 51-B, No. 6, 225-232, 1968 (in Japanese).

Mамво, M. and I. NAGANo, "Electromagnetic waves in longitudinally inhomogeneous anisotropic medium," Antenna and propagation meeting of IECE of Japan AP-71-27, 1971 (in Japanese).

Mambo, M. and S. Shimakura, Electromagnetic fields in an inhomogeneous medium of which both dielectric constant and conductivity are parabolically distributed with distance, J.I.E.C.E. of Japan, 54-B, No. 4, 139-145, 1971 (in Japanese).

Pitteway, M.L.V., The numerical calculation of wave fields, reflection coefficients and polarization for long radio waves in the lower ionosphere, I. Phil. Roy. Soc., London, A., 257, 219$241,1965$.

Whittaker, E.T. and G.N. Watson, "A course of modern analysis," Cambridge University Press, pp. 347--348, 1965. 